\title{
CARACTERIZAÇÃO E GÊNESE DO CARÁTER COESO EM LATOSSOLOS AMARELOS E ARGISSOLOS DOS TABULEIROS COSTEIROS DO ESTADO DE ALAGOAS ${ }^{(1)}$
}

\author{
José de Almeida Lima Neto ${ }^{(2)}$, Mateus Rosas Ribeiro ${ }^{(3)}$, Marcelo \\ Metri Corrêa ${ }^{(4)}$, Valdomiro Severino de Souza Júnior ${ }^{(5)}$ José \\ Fernando W.F. Lima ${ }^{(6)}$ \& Rafael Fernandes de Abreu e Lima \\ Ferreira $^{(7)}$
}

\begin{abstract}
RESUMO
O caráter coeso é um atributo característico de horizontes subsuperficiais de solos que apresentam consistência muito dura a extremamente dura, quando secos, passando a friável ou firme, quando umedecidos. A formação desses horizontes, entretanto, ainda é um assunto polêmico, não estando completamente esclarecida. Este trabalho objetivou caracterizar e identificar os possiveis mecanismos envolvidos na formação dos horizontes coesos de solos dos Tabuleiros Costeiros, buscando entender sua gênese e definir propriedades que possam complementar a definição do caráter coeso no Sistema Brasileiro de Classificação de Solos. Quatro perfis de solos da região dos Tabuleiros Costeiros de Alagoas foram estudados: um Argissolo Amarelo, um Argissolo Acinzentado e dois Latossolos Amarelos. Os solos foram descritos morfologicamente, e amostras dos horizontes coesos e não coesos
\end{abstract}

\footnotetext{
(1) Parte da Dissertação de Mestrado do primeiro autor apresentada ao programa de Pós-Graduação em Ciência de Solo, Universidade Federal Rural de Pernambuco - UFRPE. Projeto financiado pelo CNPq. Recebido para publicação em setembro de 2008 e aprovado em abril de 2009.

(2) Engenheiro-Agrônomo, Companhia Nacional de Abastecimento - CONAB, Av. Farquar 3305, Bairro Pedrinhas, CEP 7890460 Porto Velho (RO). E-mail: jdealmeida22@yahoo.com.br

(3) Professor Associado do Departamento de Agronomia, Universidade Federal Rural de Pernambuco - UFRPE. R. Dom Manoel de Medeiros s/n, Dois Irmãos, CEP 52171-900 Recife (PE). Bolsista do CNPq. E-mail: mateus.rosas@pq.cnpq.br

(4) Professor Adjunto da Unidade Acadêmica de Garanhuns, UFRPE. CEP 552986-280 Garanhuns (PE). E-mail: marcelometri@yahoo.com

(5) Professor Adjunto do Departamento de Agronomia , UFRPE. E-mail: vsouzajr@yahoo.com

(6) Engenheiro-Agrônomo do departamento de Agronomia, UFRPE. E-mail: zecasolos@yahoo.com.br

(7) Estudante de Agronomia, UFRPE. Bolsista de Iniciação Científica do CNPq. E-mail: raferural@hotamil.com
} 
foram coletadas para execução de análises físicas e químicas. A caracterização morfológica constatou a presença de horizontes com caráter coeso tanto nas áreas sob floresta quanto nas áreas sob cultivo, confirmando sua natureza pedogenética. A morfologia e a densidade do solo, aliadas à determinação da superfície específica, mostraram-se eficientes na identificação dos horizontes coesos dos solos estudados.

Termos de indexação: solos coesos, adensamento, pedogênese, Formação Barreiras.

\title{
SUMMARY: CHARACTERIZATIONAND GENESIS OFCOHESIVE HORIZONS IN OXISOLS AND ULTISOLS OF THE COASTAL TABLELANDS OF ALAGOAS STATE, BRAZIL
}

\begin{abstract}
The cohesive character is an attribute of subsurface soil horizons with hard to extremely hard consistency when dry, and friable or firm when wetted. Despite the agricultural importance, the formation of these horizons is not yet completely understood. The objective of this study was to characterize and assess the possible mechanisms involved in the formation of cohesive horizons in soils of the Coastal Tablelands of Northeast Brazil, in order to understand their pedogenesis and to identify physical and chemical parameters that can be used for the definition of the cohesive character in the Brazilian System of Soil Classification. Four soil profiles were morphologically characterized: a Yellow Argisol (Ultisol), a Gray Argisol (Ultisol) and two Yellow Latosols (Oxisols). Samples from cohesive and non-cohesive horizons were taken for physical and chemical analysis. The morphological description revealed the presence of the cohesive character both under forest as well as under cultivation, confirming its pedogenetic nature. Soil morphology and soil density, together with the specific surface area efficiently identified the cohesive character in the studied soils.
\end{abstract}

Index terms: cohesive soils, dense horizon, soil genesis; Barreiras Formation.

\section{INTRODUÇÃO}

Diferenças marcantes entre os graus de consistência do solo seco e úmido, principalmente em termos de incremento do grau de consistência do solo seco (dureza), podem ser observadas em alguns horizontes de solos da faixa dos tabuleiros costeiros, nas regiões Sudeste e Nordeste do Brasil (Giarola et al., 2001).

O termo "coeso" foi inicialmente empregado no Brasil na década de 1970 , durante a realização do levantamento exploratório de solos do Estado do Espírito Santo (Embrapa, 1978), para distinguir horizontes minerais subsuperficiais de solos que apresentavam consistência no mínimo dura, quando secos, e friável a firme, quando úmidos. Entretanto, os primeiros registros de sua identificação ocorreram na década de 1950, quando, no levantamento de solos do Estado do Rio de Janeiro, foram identificados horizontes de solos amarelos com forte resistência à penetração do martelo pedológico (Barros et al., 1958).

Esse tipo de comportamento do solo foi incorporado ao Sistema Brasileiro de Classificação de Solos (SiBCS) por meio da criação e utilização do atributo diagnóstico "caráter coeso" (Embrapa, 2006). Esse caráter é uma característica pedogenética (adensamento), típica de horizontes subsuperficiais (BA e, ou, parte do Bw ou
Bt), de textura média, argilosa ou muito argilosa, encontrada normalmente entre 0,30 e $0,70 \mathrm{~m}$ de profundidade, muito comum em solos dos sedimentos terciários da Formação Barreiras. Em condições naturais, apresentam uma fraca organização estrutural e são geralmente maciços, com consistência muito dura ou extremamente dura quando secos, passando a friáveis ou firmes quando úmidos.

Os solos coesos estão intimamente relacionados com depósitos sedimentares de textura média a argilosa, muito intemperizados, referidos ao período terciário, representados pela Formação Barreiras. A mineralogia da fração argila desses solos apresenta a caulinita como mineral dominante, e a fração areia é constituída essencialmente por quartzo (Rezende, 2000). Em função do material de origem, os teores de $\mathrm{Fe}_{2} \mathrm{O}_{3}$ (ataque com $\mathrm{H}_{2} \mathrm{SO}_{4}$ ) são inferiores a $80 \mathrm{~g} \mathrm{~kg}^{-1}$, com predomínio de goethita, o que confere aos solos as cores amareladas típicas, geralmente bruno-amarelada, tendendo muitas vezes para cores mais pálidas, brunoamarelado-claro, bruno-claro-acinzentado ou bruno no matiz 10 YR, com valores 5 e 6 e cromas entre 3 e 6 (Ribeiro, 2001). Uma característica comum desses solos é o Ki elevado (normalmente de 1,7 a 2,0), quando comparado aos valores geralmente observados nos Latossolos, em função da presença dominante de caulinita. 
Os solos dos tabuleiros costeiros ocupam, na região Nordeste, uma área estimada de 10.000.000 ha, o que corresponde a aproximadamente $16 \%$ da área total da Bahia, Sergipe, Alagoas, Pernambuco, Paraíba, Rio Grande do Norte e Ceará (Souza et al., 2001). Essas áreas são de grande importância socioeconômica, devido à proximidade dos grandes centros urbanos, sendo utilizadas com a cultura da cana-de-açúcar ou dedicadas à produção de alimentos. Nos Estados de Alagoas, Pernambuco, Paraíba e Rio Grande do Norte, o uso atual dominante é a cultura da cana-de-açúcar.

A formação dos horizontes com caráter coeso ainda é um assunto polêmico, não estando completamente esclarecida. Sabe-se, porém, que ocorre de forma natural e que pode estar associada a vários processos, como: entupimento dos poros com argila iluvial; presença de compostos orgânicos poucos polimerizados; presença e acúmulo de sílica secundária, óxido de Fe e argila dispersa nos microporos; e adensamento por dessecação resultante da alteração da estrutura do solo pela alternância de ciclos de umedecimento e secagem.

O objetivo deste trabalho foi proceder à caracterização morfológica, física e química de horizontes coesos e não coesos em Latossolos e Argissolos, do ambiente dos tabuleiros costeiros, buscando entender sua gênese e identificar propriedades físicas e químicas, que possam complementar a definição do caráter coeso, baseada exclusivamente em características morfológicas.

\section{MATERIAL E MÉTODOS}

Foram descritos e coletados, conforme Santos et al. (2005), quatro perfis de solos com expressiva manifestação do caráter coeso: um Argissolo Amarelo distrocoeso fragipânico (PA, Perfil 1), um Latossolo Amarelo distrocoeso típico (LA1, Perfil 2), um Argissolo Acinzentado distrocoeso fragipânico (PAC, Perfil 3), e um Latossolo Amarelo distrocoeso típico (LA2, Perfil 4).

Os solos foram coletados na zona do litoral e mata do Estado de Alagoas, na mesorregião leste alagoano (Mendes Júnior, 2002), sendo as coletas realizadas na Usina Coruripe, no município de Coruripe (PA, Perfil 1), na Usina Caeté, em São Miguel dos Campos (LA1, Perfil 2), na Usina Marituba, em Penedo (PAC, Perfil 3), e na Usina Cachoeira, no município de Maceió (LA2, Perfil 4) (Figura 1).

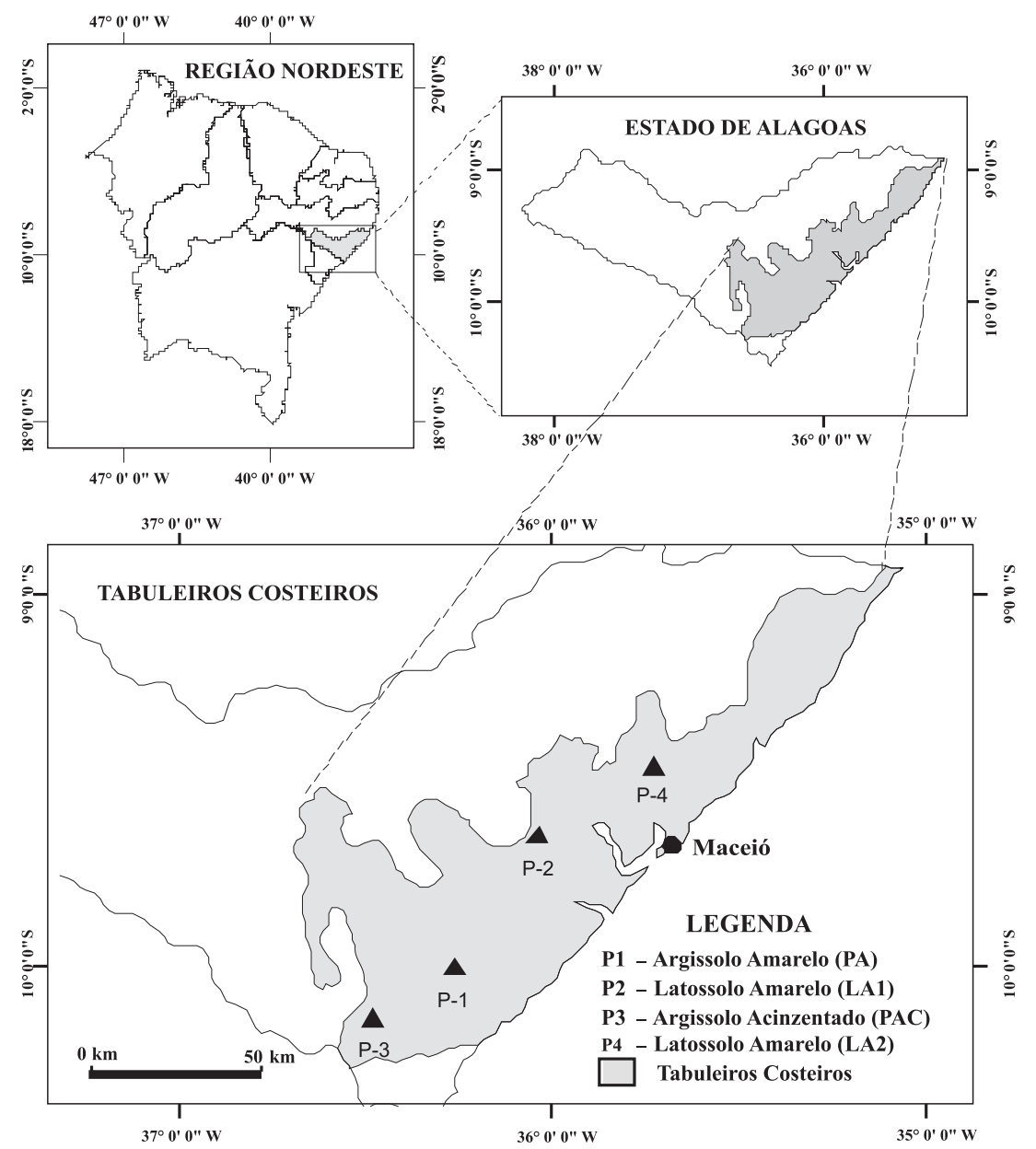

Figura 1. Localização das áreas de coleta dos solos. 
A área de coleta dos solos apresenta clima tropical chuvoso com verão seco (As', segundo a classificação de Köppen). A precipitação pluvial média anual nos municípios onde os solos foram coletados fica em torno de $1.400 \mathrm{~mm}$ em Coruripe, $1.650 \mathrm{~mm}$ em Penedo e São Miguel dos Campos, e $1.850 \mathrm{~mm}$ em Maceió. A temperatura média anual da região está em torno de $25,5^{\circ} \mathrm{C}$. O relevo da região é predominantemente plano na superfície dos tabuleiros, que são entalhados por vales profundos. A vegetação primária da região era constituída pela floresta subperenifólia, formação dominante na zona úmida costeira do Estado, que cobria extensas áreas dos tabuleiros, bem como do cristalino, estando hoje resumida a pequenas áreas restritas a encostas e pequenas áreas de preservação nos topos dos tabuleiros (Jacomine et al., 1975).

Os dois primeiros perfis (PA e LA1) foram coletados sob vegetação nativa. Já os demais foram coletados em áreas cultivadas com cana-de-açúcar. A coleta foi realizada por horizontes, para a realização das análises químicas de rotina, além do ataque sulfúrico.

Visando dar mais detalhe às propriedades relacionadas com o caráter coeso, e aferir a exatidão da descrição morfológica na delimitação desses horizontes, as amostras para as determinações físicas foram coletadas de $10 \mathrm{em} 10 \mathrm{~cm}$, a partir do horizonte que precede os horizontes definidos morfologicamente como coesos, até o fim do horizonte subjacente, exceto quando o horizonte de caráter coeso transitava de forma abrupta para um horizonte fragipã (Argissolos).

As análises físicas e químicas foram realizadas segundo Embrapa (1997). Os elementos Si, Al, Fe foram determinados na fração TFSA após ataque sulfúrico, segundo Embrapa (1997) e conforme modificações sugeridas por Corrêa (2000). A superfície específica (SE) foi determinada na fração argila de todos os horizontes coletados, pelo método da sorção de vapor de água (Quirk, 1955). A fração argila foi obtida por sedimentação, após extração da matéria orgânica com hipoclorito de sódio e dispersão com $\mathrm{NaOH} 0,01 \mathrm{~mol} \mathrm{~L}^{-1}$ (Jackson, 1979).

\section{RESULTADOS E DISCUSSÃO}

\section{Caracterização morfológica}

Foram observadas variações de cor, consistência e estrutura entre os horizontes e entre os solos. Os perfis mostraram sequência de horizontes $\mathrm{A}, \mathrm{AB}, \mathrm{BA}$ e Bt nos Argissolos, e A, $\mathrm{AB}$ e, ou, BA e Bw nos Latossolos, todos muito profundos (Quadro 1).

Os solos apresentam cores predominantemente amareladas nas matrizes 10YR e 7,5 YR, com exceção do PAC, que é, predominantemente acinzentado, com matiz 10 YR, valores $\geq 5$ e cromas $<4$ na maioria dos primeiros $100 \mathrm{~cm}$ do horizonte $\mathrm{B}$, indicando má drenagem. Os fatores que condicionam a má drenagem desse solo são a posição levemente mais baixa na paisagem e a presença de horizonte fragipã em subsuperfície.

Foram observados mosqueados pequenos e distintos nos horizontes coesos, com exceção do Argissolo Acinzentado (PAC). Nos fragipãs dos perfis PA e PAC, também foram observados mosqueados em maior quantidade e mais contrastantes do que os observados nos horizontes coesos. Os mosqueados vermelhos dos horizontes Bt/Btx do Argissolo Acinzentado, visualmente muito semelhantes à plintita, numa observação mais detalhada em volume, foram identificados como revestimentos de óxidos de $\mathrm{Fe}$ (filmes) em alguns planos de fraqueza.

A descrição morfológica dos perfis evidenciou, através das marcantes diferenças nos graus de consistência de solos seco e úmido, e da presença de estrutura maciça coesa, horizontes com caráter coeso em todos os perfis, tanto nas áreas sob floresta quanto nas áreas sob cultivo, confirmando sua natureza pedogenética. A consistência é extremamente dura e firme nos fragipãs. Os solos apresentaram ainda uma consistência plástica e pegajosa nos horizontes coesos, com exceção do horizonte BA do perfil PA, que se apresentou ligeiramente plástico e ligeiramente pegajoso.

O caráter coeso está presente nos horizontes BA, podendo se estender para o $\mathrm{Bt}$ (perfil $\mathrm{PA}$ ), $\mathrm{AB}$ (perfil PAC) e Bw1 (perfil LA2), dentro da profundidade de cada um (destacados em negrito no Quadro 1). Segundo Jacomine (1996) e Ribeiro (2001), esse caráter ocorre em condições naturais, principalmente nos horizontes transicionais $\mathrm{AB}$ e, ou, $\mathrm{BA}$, podendo atingir o Bw ou Bt, corroborando a descrição de campo. Nos solos cultivados com cana-de-açúcar (PAC e LA2), foram observados horizontes endurecidos mais superficiais, com consistência muito dura, concordando com Silva \& Ribeiro (1992). Segundo esses autores, em áreas de cultivo contínuo de canade-açúcar, o maior grau de coesão, com aumento da densidade do solo, foi constatado mais próximo da superfície, como consequência de alterações promovidas pelo uso e manejo (compactação).

Os horizontes coesos apresentaram uma significativa redução na quantidade e no diâmetro das raízes, variando de poucas a raras. Os perfis PA e LA2 apresentaram raízes em quantidade comum no topo dos horizontes coesos. A elevada resistência à penetração desses horizontes quando secos dificulta $o$ crescimento das raízes, que tendem a se concentrar na superfície. Souza (1996) afirma que, apesar de serem considerados profundos, os solos de tabuleiro possuem uma profundidade efetiva reduzida pela presença desses horizontes.

A elevada resistência à penetração das raízes nos horizontes coesos foi bem caracterizada na morfologia do Argissolo Acinzentado, pois o Ap apresenta muitas 


\section{Quadro 1. Características morfológicas dos solos estudados ${ }^{(1)}$}

\begin{tabular}{|c|c|c|c|c|c|c|c|c|}
\hline \multirow{2}{*}{ Horizonte } & \multirow{2}{*}{ Profundidade } & \multirow{2}{*}{ Cor (úmido) } & \multirow{2}{*}{ Mosqueado } & \multirow{2}{*}{ Estrutura } & \multicolumn{3}{|c|}{ Consistência } & \multirow{2}{*}{$\begin{array}{l}\text { Transpo- } \\
\text { sição }\end{array}$} \\
\hline & & & & & Seca & Úmida & Molhada & \\
\hline \multicolumn{9}{|c|}{ Argissolo Amarelo distrocoeso abrupto fragipânico (PA - Perfil 1) - Mata } \\
\hline $\mathrm{A}$ & $0-18$ & $10 \mathrm{YR} 4 / 2$ & - & $1 \mathrm{P} M \mathrm{Gr}$ & Ma LD & $\mathrm{MFr}$ & LgPl LgPe & gp \\
\hline $\mathrm{AB}$ & $18-40$ & $10 \mathrm{YR} 4 / 3$ & - & $1 \mathrm{P} \mathrm{M} \mathrm{Bls}$ & $\mathrm{LD}$ & $\mathrm{MFr}$ & LgPl LgPe & gp \\
\hline $\mathrm{BA}$ & $40-59$ & 10YR 5/3 & - & 1 P M Bls & MD & $\mathbf{F r}$ & LgPl LgPe & cp \\
\hline $\mathrm{Bt}$ & $59-90$ & $10 \mathrm{YR} 5 / 3$ & c m di $7,5 Y R$ 5/4 & 1 Mc Mco P M Bls & MD & $\mathbf{F i}$ & $\mathrm{Pl} \mathbf{P e}$ & ai \\
\hline Btx & $90-150+$ & $10 \mathrm{YR} 5 / 6$ & c pe pr 5 YR $4 / 6$ & Mc coesa & $\mathrm{ED}$ & Mfi & LgPl LgPe & - \\
\hline \multicolumn{9}{|c|}{ Latossolo Amarelo distrocoeso típico (LA1 - Perfil 2) - Mata } \\
\hline $\mathrm{A}$ & $0-18$ & 10YR $3 / 2$ & - & $2 \mathrm{P} \mathrm{M} \mathrm{Gr}$ & LD & $\mathrm{MFr}$ & LgPl LgPe & ao \\
\hline $\mathrm{AB}$ & $18-35$ & $10 Y R \quad 4 / 3$ & - & $1 \mathrm{P} \mathrm{M} \mathrm{Bls}$ & $\mathrm{D}$ & $\mathrm{Fr}$ & LgPl LgPe & $\mathrm{cp}$ \\
\hline $\mathrm{BA}$ & $35-55$ & 10YR 5/4 & p pe di 7,5 YR $5 / 6$ & Mc MCo & MD & $\mathbf{F i}$ & $\mathrm{Pl} \mathrm{Pe}$ & gp \\
\hline $\mathrm{Bw} 1 *$ & $55-100$ & $10 Y R 5 / 6$ & - & $1 \mathrm{P} \mathrm{M} \mathrm{Bls}$ & $\mathrm{MD}$ & $\mathrm{Fr}$ & $\mathrm{Pl} \mathrm{Pe}$ & $\mathrm{dp}$ \\
\hline $\mathrm{Bw} 2$ & $100-145$ & 10YR 5/8 & - & 1MP M Bls AMc & D MD & $\mathrm{Fr}$ & $\mathrm{Pl} \mathrm{Pe}$ & gp \\
\hline Bw3 & $145-160+$ & 10YR $6 / 8$ & - & $1 \mathrm{MP} \mathrm{Bls} \mathrm{Amc}$ & $\mathrm{D}$ & $\mathrm{Fr}$ & $\mathrm{Pl} \mathrm{Pe}$ & - \\
\hline \multicolumn{9}{|c|}{ Argissolo Acinzentado distrocoeso fragipânico (PAC - Perfil 3) - Cana } \\
\hline Ap1 & $0-11$ & 10YR 4/1 & - & Mc Mco 1P M Gr Bls & $\mathrm{MD}$ & $\mathrm{Fr}$ & LgPl LgPe & $\mathrm{cp}$ \\
\hline Ap2 & $11-27$ & $10 \mathrm{YR} 4 / 2$ & - & Mc Mco 1 P M Bls & $\mathrm{MD}$ & $\mathrm{Fr}$ & LgPl LgPe & $\mathrm{cp}$ \\
\hline $\mathrm{AB}$ & $27-42$ & $10 \mathrm{YR} 5 / 2$ & - & Mc Co & ED & $\mathrm{Fi}$ & $\mathrm{Pl} \mathbf{P e}$ & gp \\
\hline $\mathrm{BA}$ & $42-65$ & 10YR 6/3 & - & Mc Co & ED & $\mathbf{F i}$ & $\mathbf{P l} \mathbf{P e}$ & gp \\
\hline Bt & $65-90$ & $10 \mathrm{YR} 6 / 3$ & $\mathrm{c} \mathrm{m}$ di $10 \mathrm{YR} 7 / 3$ & Mc Co 1 P M Bls & $\mathrm{D} \mathrm{MD}$ & Fr e Fi & $\mathrm{Pl} \mathrm{Pe}$ & $\mathrm{ci}$ \\
\hline $\mathrm{Bt} / \mathrm{Btx} 1$ & $90-130$ & 10YR $6 / 3$ & $\begin{array}{l}\text { c m pr } 2,5 Y R \quad 4 / 6 \text { e c m } \\
\text { di } 10 Y R ~ 7 / 4\end{array}$ & $1 \mathrm{P} \mathrm{M} \mathrm{Bls}$ & D ED & Fr MFi & $\mathrm{Pl} \mathrm{Pe}$ & co \\
\hline $\mathrm{Bt} / \mathrm{Btx} 2$ & $130-160+$ & 10YR $6 / 3$ & $\begin{array}{l}\text { p pe m pr } 2,5 Y R \quad 4 / 6 \text { e } \\
\text { c m gr di } 10 Y R ~ 7 / 4\end{array}$ & $1 \mathrm{P} \mathrm{M} \mathrm{Bls}$ & D ED & Fr MFi & $\mathrm{Pl} \mathrm{Pe}$ & - \\
\hline \multicolumn{9}{|c|}{ Latossolo Amarelo distrocoeso típico (LA2 - Perfil 4) - Cana } \\
\hline Ap & $0-22$ & $10 \mathrm{YR} 4 / 2$ & - & Mc Mco 1 P M Bls & $\mathrm{MD}$ & $\mathrm{Fr}$ & $\mathrm{Pl} \mathrm{Pe}$ & ao \\
\hline $\mathrm{BA}$ & $22-40$ & 10YR 5/6 & p pe di 5 YR 5/6 & Mc Mco 1 P M Bls & MD & $\mathbf{F i}$ & $\mathrm{Pl} \mathrm{Pe}$ & gp \\
\hline Bw1 & $40-65$ & $10 \mathrm{YR} 5 / 8$ & - & 1 P M Bls CPMa & MD & $\mathbf{F i}$ & $\mathrm{Pl} \mathrm{Pe}$ & gp \\
\hline $\mathrm{Bw} 2$ & $65-110$ & 10 YR $5 / 8$ & - & $1 \mathrm{MP} P \mathrm{Bls}$ & $\mathrm{D}$ & $\mathrm{Fr}$ & $\mathrm{Pl} \mathrm{Pe}$ & $\mathrm{dp}$ \\
\hline Bw3 & $110-170+$ & $7,5 \mathrm{YR} 5 / 8$ & - & 1 MP P Bls CPMp & $\mathrm{D}$ & $\mathrm{Fr}$ & $\mathrm{Pl} \mathrm{Pe}$ & - \\
\hline
\end{tabular}

(1) Os resultados em negrito correspondem aos horizontes coesos identificados na descrição morfológica. Estrutura: 1: fraca; 2: moderada; MP: muito pequena; P: pequena; M: média; Gr: granular; Bls: blocos angulares; Bls: blocos subangulares; Mc: maciça; Mco: moderadamente coesa; Co: coesa. Consistência: Ma: macia; LD: ligeiramente dura; D: dura; MD: muito dura; ED: extremamente dura; MFr: muito friável; Fr: friável; Fi: firme; MFi: muito firme; Lg: ligeiramente; Pl: plástica; Pe: pegajosa. Transição: p: plana; o: ondulada; a: abrupta; g: gradual; d: difusa; c: clara; i: irregular; Mosqueado: p: pouco; c: comum; m: médio; pe: pequeno; di: distinto; pr: proeminente. ${ }^{*} \mathrm{O}$ topo deste horizonte mostra-se coeso.

raízes, passando para poucas no $\mathrm{AB}$, raras no $\mathrm{BA}$, ambos coesos, aumentando para comuns no horizonte Bt. No perfil LA2 ocorre situação semelhante, sendo comuns as raízes nos horizontes $\mathrm{BA}$ e $\mathrm{Bw}_{3}$, e poucas no coeso $\mathrm{Bw}_{1}$. A passagem das raízes pelo horizonte coeso pode ocorrer através de espaços descontínuos não coesos ou através das raízes de plantas com maior poder de penetração. A penetração inicial de raízes promovida por plantas mais adaptadas abriria caminho para o sistema radicular de outras espécies atravessar o horizonte coeso.

Nos perfis sob vegetação primária, observou-se um ligeiro aumento de raízes nos horizontes coesos em relação aos cultivados com cana, devido ao maior poder de penetração e das raízes pivotantes da vegetação primária.
Os horizontes coesos apresentam estrutura maciça coesa e, ou, moderadamente coesa, com ou sem partes com estrutura fraca pequena a média em blocos subangulares. Perfis descritos na Reunião Técnica sobre Solos Coesos de Tabuleiros Costeiros, em 1996, na Bahia, mostram solos coesos com estrutura fraca pequena e média em blocos subangulares, associada a fragmentos coesos. Esses casos podem ser explicados por áreas com maior concentração de matéria orgânica (Silva \& Ribeiro, 1992).

\section{Caracterização física}

Para todos os perfis, as análises granulométricas revelaram uma textura média em superfície, passando para argilosa em profundidade (Quadro 2). Nos Latossolos, há uma transição gradual para a textura 
Quadro 2. Características físicas dos solos estudados ${ }^{(1)}$

\begin{tabular}{|c|c|c|c|c|c|c|c|c|c|c|c|c|}
\hline \multirow{2}{*}{ Horiz. } & \multirow{2}{*}{ Camada } & \multicolumn{2}{|c|}{ Areia } & \multirow{2}{*}{ Silte } & \multirow{2}{*}{ Argila } & \multirow{2}{*}{ AD água } & \multirow{2}{*}{ GF } & \multirow{2}{*}{ Silte/argila } & \multirow{2}{*}{ Ds } & \multirow{2}{*}{ Dp } & \multirow{2}{*}{ Porosidade } & \multirow{2}{*}{ SE } \\
\hline & & Grossa & Fina & & & & & & & & & \\
\hline & $\mathrm{cm}$ & & 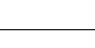 & $-\mathrm{g} \mathrm{kg}^{-1}$ & 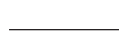 & - & $\%$ & & $-\mathrm{kg}$ & $m^{-3}-$ & $\%$ & $m^{2} g^{-1}$ \\
\hline & & & Argi & ssolo $\mathrm{Ar}$ & narelo dis & strocoeso al & rupto $f$ & $\mathrm{ra}$ & Perfil & - Mat & & \\
\hline $\mathrm{A}$ & $0-18$ & 567 & 233 & 60 & 140 & 55 & 61 & 0,43 & 1,26 & 2,66 & 53 & 29,73 \\
\hline $\mathrm{AB}$ & $30-40$ & 497 & 206 & 75 & 222 & 89 & 53 & 0,56 & 1,57 & 2,66 & 41 & 29,63 \\
\hline \multirow{2}{*}{$\mathrm{BA}$} & $40-50$ & 466 & 210 & 93 & 231 & 180 & 31 & 0,40 & 1,52 & 2,63 & 42 & 27,53 \\
\hline & $50-60$ & 370 & 175 & 96 & 359 & 229 & 36 & 0,27 & 1,51 & 2,63 & 42 & 29,37 \\
\hline \multirow[t]{2}{*}{$\mathrm{Bt}$} & $60-70$ & 356 & 156 & 57 & 431 & 304 & 29 & 0,13 & 1,49 & 2,63 & 43 & 29,85 \\
\hline & $70-80$ & 296 & 139 & 62 & 503 & 335 & 33 & 0,12 & 1,54 & 2,61 & 41 & 29,18 \\
\hline \multirow[t]{2}{*}{ Btx } & $90-150$ & 200 & 112 & 110 & 578 & 340 & 43 & 0,16 & 1,54 & 2,58 & 40 & 29,18 \\
\hline & \multicolumn{12}{|c|}{ Latossolo Amarelo distrocoeso típico (LA1 - Perfil 2) - Mata } \\
\hline A & $0-18$ & 530 & 153 & 82 & 235 & 132 & 43 & 0,35 & 1,46 & 2,55 & 43 & 34,87 \\
\hline \multirow[t]{2}{*}{$\mathrm{AB}$} & $18-28$ & 492 & 160 & 39 & 309 & 145 & 53 & 0,13 & 1,47 & 2,59 & 43 & 34,55 \\
\hline & $28-38$ & 457 & 147 & 39 & 357 & 206 & 41 & 0,11 & 1,54 & 2,59 & 41 & 33,35 \\
\hline \multirow[t]{2}{*}{$\mathrm{BA}$} & $38-48$ & 398 & 138 & 29 & 435 & 290 & 33 & 0,07 & 1,54 & 2,59 & 41 & 30,30 \\
\hline & $48-58$ & 352 & 122 & 42 & 484 & 300 & 38 & 0,09 & 1,45 & 2,59 & 44 & 35,61 \\
\hline \multirow[t]{4}{*}{ Bw1 } & $58-68$ & 325 & 114 & 70 & 491 & 234 & 52 & 0,14 & 1,49 & 2,59 & 42 & 35,70 \\
\hline & $68-78$ & 330 & 110 & 79 & 481 & 99 & 79 & 0,16 & 1,43 & 2,59 & 45 & 34,89 \\
\hline & $78-88$ & 342 & 118 & 36 & 504 & 0 & 100 & 0,05 & 1,41 & 2,59 & 46 & 36,41 \\
\hline & $88-98$ & 312 & 110 & 30 & 548 & 0 & 100 & 0,04 & 1,43 & 2,59 & 45 & 34,84 \\
\hline Bw2 & $100-145$ & 314 & 125 & 28 & 533 & 0 & 100 & 0,03 & 1,40 & 2,59 & 46 & 33,67 \\
\hline Bw3 & $145-160$ & 233 & 95 & 33 & 639 & 0 & 100 & 0,05 & 1,39 & 2,59 & 46 & 31,03 \\
\hline & & & & sol & inzent & ado distro & so fra & ânico (PAC & Perfil : & - Cana & & \\
\hline Ap1 & $0-11$ & 340 & 264 & 89 & 307 & 185 & 42 & 0,33 & 1,59 & 2,56 & 38 & 24,48 \\
\hline Ap2 & $15-25$ & 320 & 257 & 76 & 347 & 220 & 44 & 0,27 & 1,73 & 2,56 & 32 & 26,48 \\
\hline $\mathrm{AB}$ & $25-35$ & 258 & 231 & 74 & 436 & 302 & 37 & 0,10 & 1,68 & 2,63 & 36 & 26,93 \\
\hline & $35-45$ & 219 & 219 & 71 & 489 & 301 & 46 & 0,08 & 1,67 & 2,63 & 37 & 28,00 \\
\hline BA & 45-55 & 180 & 197 & 95 & 528 & 0 & 100 & 0,12 & 1,68 & 2,63 & 36 & 29,93 \\
\hline & $55-65$ & 189 & 194 & 87 & 530 & 0 & 100 & 0,09 & 1,54 & 2,63 & 41 & 30,04 \\
\hline $\mathrm{Bt}$ & $65-75$ & 171 & 194 & 95 & 540 & 0 & 100 & 0,09 & 1,53 & 2,63 & 42 & 28,05 \\
\hline & $75-85$ & 166 & 190 & 99 & 545 & 0 & 100 & 0,14 & 1,41 & 2,63 & 46 & 26,88 \\
\hline $\mathrm{Bt} / \mathrm{Btx} 1$ & $90-130$ & 114 & 132 & 159 & 595 & 13 & 98 & 0,14 & 1,56 & 2,55 & 39 & 25,26 \\
\hline $\mathrm{Bt} / \mathrm{Btx} 2$ & $130-160$ & 90 & 124 & 191 & 592 & 0 & 100 & 0,28 & & 2,56 & - & 27,56 \\
\hline & & & & Latos: & solo Amar & relo distroc & eso típ & ico (LA2 - Perfi & 4) - $\mathrm{Ca}$ & & & \\
\hline Ap & $0-22$ & 354 & 188 & 117 & 341 & 256 & 18 & 0,46 & 1,63 & 2,59 & 37 & 32,77 \\
\hline BA & $22-32$ & 293 & 143 & 91 & 473 & 390 & 20 & 0,12 & 1,58 & 2,63 & 40 & 35,47 \\
\hline & $32-42$ & 258 & 143 & 49 & 550 & 442 & 25 & 0,03 & 1,55 & 2,63 & 41 & 36,01 \\
\hline Bw1 & $42-52$ & 256 & 128 & 37 & 579 & 463 & 26 & 0,05 & 1,50 & 2,63 & 43 & 33,20 \\
\hline & $52-62$ & 265 & 123 & 31 & 581 & 471 & 23 & 0,03 & 1,46 & 2,63 & 44 & 35,38 \\
\hline Bw2 & $62-72$ & 265 & 129 & 24 & 582 & 0 & 100 & 0,04 & 1,45 & 2,56 & 43 & 35,04 \\
\hline & $72-82$ & 265 & 128 & 23 & 584 & 0 & 100 & 0,03 & 1,36 & 2,59 & 47 & 36,28 \\
\hline & $82-92$ & 248 & 135 & 32 & 585 & 0 & 100 & 0,03 & 1,23 & 2,59 & 53 & 35,06 \\
\hline & $92-102$ & 254 & 134 & 31 & 581 & 0 & 100 & 0,05 & 1,24 & 2,63 & 53 & 36,28 \\
\hline & $102-112$ & 235 & 130 & 41 & 594 & 0 & 100 & 0,08 & 1,25 & 2,59 & 52 & 33,42 \\
\hline Bw3 & $110-170$ & 210 & 102 & 83 & 605 & 0 & 100 & 0,15 & 1,23 & 2,63 & 53 & 32,00 \\
\hline
\end{tabular}

(1) Os resultados em negrito correspondem aos horizontes coesos identificados na descrição morfológica. Hor.: horizonte; Cam. camada; PT: porosidade total; SE: superfície específica $\mathrm{em}^{2}$ por grama de argila.

argilosa, e os dois perfis atingem textura muito argilosa no $\mathrm{Bw}_{3}$, sem apresentar, entretanto, gradiente suficiente para caracterizar um B textural.

No perfil PA, o teor de argila de $578 \mathrm{~g} \mathrm{~kg}^{-1}$ no fragipã é bem superior aos $350 \mathrm{~g} \mathrm{~kg}^{-1}$ sugerido por Petersen et al. (1970) e Richie et al (1974) como limite máximo para formação desses horizontes. Segundo Corrêa (2005), a mineralogia caulinítica dos solos de tabuleiros e a alta relação argila fina/argila grossa são os principais motivos para ocorrência do fragipã de textura argilosa.

Todos os perfis estudados apresentaram baixos teores de silte. De acordo com Moreau et al. (2006), os baixos valores da relação silte/argila dos Argissolos Amarelos devem-se ao fato de ser produto da alteração de sedimentos pré-intemperizados e edafizados. Os maiores valores da relação silte/argila foram observados nos horizontes superficiais devido, 
provavelmente, à perda relativa de argila na superfície por eluviação ou dissolução (Silva et al., 2002). Os horizontes coesos estudados apresentaram textura franco-argilo-arenosa, argilo-arenosa e argilosa, como relatado por outros autores (Moreau, 2001, Corrêa, 2005).

A densidade do solo vem corroborar os resultados morfológicos obtidos em campo. O horizonte coeso do Argissolo Acinzentado apresentou a maior densidade do solo entre os coesos estudados $\left(1,68 \mathrm{~kg} \mathrm{dm}^{-3}\right)$ e uma estrutura maciça coesa, com consistência extremamente dura. Os horizontes coesos que apresentaram menor densidade do solo, em relação ao Argissolo Acinzentado, apresentaram estrutura maciça moderadamente coesa e consistência muito dura e, em alguns casos, apresentaram estrutura maciça e fraca pequena a média em blocos subangulares, como nos perfis PA e LA2. Os maiores valores de densidade do solo dos horizontes coesos, em relação aos demais horizontes do perfil (Quadro 2), sugerem que este atributo é um importante indicador para avaliar a presença de coesão.

A densidade dos horizontes coesos encontra-se na faixa de 1,50 a 1,68 $\mathrm{kg} \mathrm{dm}^{-3}$, com média de $1,59 \mathrm{~kg} \mathrm{dm}^{-3}$, estando de acordo com outros autores (Araújo Filho et al., 2001; Corrêa, 2005) que estudaram solos coesos desenvolvidos de sedimentos da Formação Barreiras.

Nos perfis localizados sob mata nativa, a densidade do solo foi maior no horizonte coeso (BA), o que não ocorreu nos solos sob o cultivo de cana-de-açúcar (PAC e LA2). Na condição de cultivo, os perfis apresentam aumentos significativos nos valores de densidade do solo nos horizontes superficiais $\left(1,73 \mathrm{~kg} \mathrm{dm}^{-3}\right.$ no horizonte Ap2 do perfil PAC). Silva (1996) constatou que, sob mata, a densidade do solo foi maior no BA que nos demais horizontes. O mesmo autor afirmou que o adensamento do horizonte BA é de origem genética, e a drástica redução da porosidade é consequência do preenchimento de poros com argila iluvial.

O perfil PA apresentou, no fragipã, densidade do solo similar à encontrada no horizonte coeso do mesmo perfil, portanto apresenta elevada densidade desde os primeiros 30 centímetros até $150 \mathrm{~cm}$ de profundidade. Neste perfil, o horizonte coeso estende-se desde os $40 \mathrm{~cm}$ de profundidade até os $90 \mathrm{~cm}$, apresentando $50 \mathrm{~cm}$ de espessura, o mais espesso entre os solos estudados. De acordo com Jacomine (1996), os Argissolos Amarelos com mudança textural mais pronunciada apresentam o caráter coeso até maiores profundidades.

A argila dispersa em água apresentou os valores mais elevados no fragipã do PA e nos horizontes coesos, sendo observado um grau de floculação mais baixo em relação aos outros horizontes (Quadro 2). Resultados semelhantes foram obtidos por Moreau (2001), Barzegar et al. (1995) e Corrêa et al. (2008), que descreveram uma relação positiva entre o teor de argila dispersa em água e a resistência à penetração.
A superfície específica (SE) apresentou valores variando de 24 a $36 \mathrm{~m}^{2} \mathrm{~g}^{-1}$ de argila. Os maiores valores foram observados nos horizontes coesos de todos os perfis estudados, sugerindo maior proporção de argilas muito finas, que, segundo Corrêa (2005), pode contribuir para o desenvolvimento do caráter coeso. Em estudos desenvolvidos por esse autor, o aumento na relação argila fina/argila grossa nos horizontes coesos de Argissolos e Latossolos está relacionado com a forte resistência à penetração e às demais características morfológicas desses horizontes.

Com relação à porosidade total, o perfil LA1, por estar em área preservada, é o solo que melhor caracteriza a presença do caráter coeso. Neste perfil, os horizontes coesos apresentam valores inferiores de porosidade em relação aos demais horizontes. Dessa forma, o horizonte coeso reduz a infiltração da água no solo causando restrição de aeração e promovendo o acúmulo de água em depressões, e o aumento do escoamento superficial da água da chuva ou irrigação (Mullins, 1997).

No Argissolo Amarelo, a condutividade hidráulica saturada $\left(\mathrm{K}_{\mathrm{sat}}\right)$ é alta em superfície, de $85,83 \mathrm{~cm} \mathrm{~h}^{-1}$, decrescendo acentuadamente em profundidade, devido à presença do horizonte coeso (BA e Bt), seguido pelo fragipã, que se apresenta bem mais impermeável em relação ao horizonte coeso, com uma $\mathrm{K}_{\text {sat }}$ próxima de zero $\left(0,48 \mathrm{~cm} \mathrm{~h}^{-1}\right)$ (Figura 2).

A baixa condutividade hidráulica saturada no horizonte coeso é bem visível no Latossolo Amarelo (LA1), apresentando-se alta em superfície $\left(50,87 \mathrm{~cm} \mathrm{~h}^{-1}\right)$, com redução considerável no horizonte coeso BA $\left(10,02 \mathrm{~cm} \mathrm{~h}^{-1}\right)$, e aumentando novamente no horizonte $\mathrm{Bw}_{3}\left(\sim 55 \mathrm{~cm} \mathrm{~h}^{-1}\right)$.

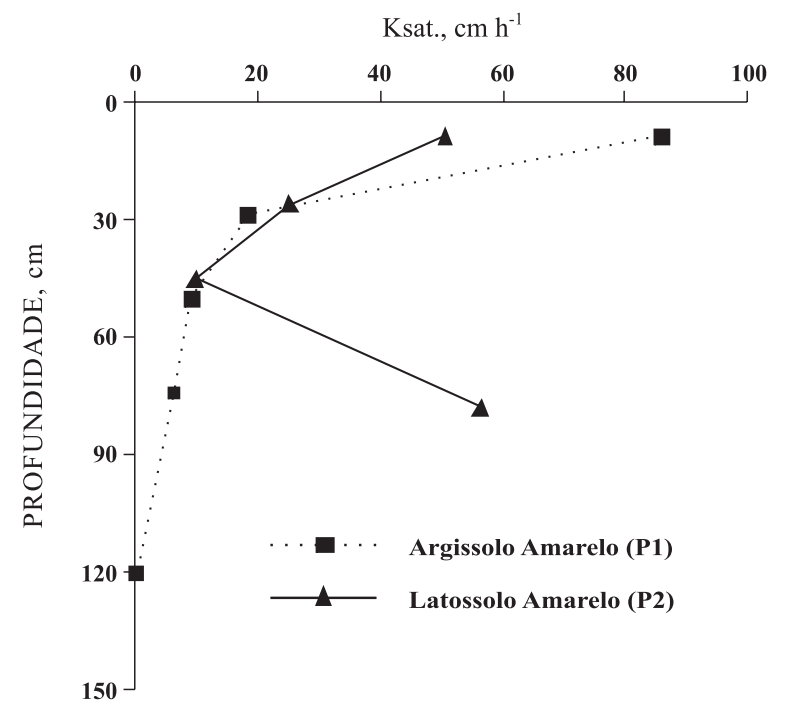

Figura 2. Condutividade hidráulica saturada $\left(\mathrm{K}_{\mathrm{sat}}\right)$ em Argissolo Amarelo (PA) e Latossolo Amarelo (LA1) dos tabuleiros costeiros de Alagoas, sob mata nativa. 


\section{Caracterização química Análises de rotina}

Os solos apresentaram limitada disponibilidade de nutrientes, com baixos valores de soma de bases (S), capacidade de troca de cátions (CTC) e elevados teores de alumínio trocável $\left(\mathrm{Al}^{3+}\right)$ e saturação por $\mathrm{Al}^{3+}(\mathrm{m})$ (Quadro 3). Os baixos valores de CTC, inferiores a $7,9 \mathrm{cmol}_{\mathrm{c}} \mathrm{kg}^{-1}$, refletem a mineralogia caulinítica do material de origem dos solos. Tais resultados corroboram os encontrados por vários autores (Jacomine, 1996; Silva \& Ribeiro, 1998), que afirmam que os solos de tabuleiros costeiros são quimicamente pobres por se desenvolverem de materiais altamente intemperizados.

Os perfis estudados apresentam $\mathrm{pH}$ ácido, associado à saturação por bases dos horizontes superficiais, abaixo de $39 \%$, com exceção do perfil LA2, cujos valores foram superiores a $88 \%$, devido à aplicação recente de calcário. No horizonte $\mathrm{B}$, sem a influência da adição dos insumos agrícolas, a saturação por bases variou entre 4 e $18 \%$ em todos os perfis. Os maiores valores de saturação por $\mathrm{Al}^{3+}$ foram encontrados no perfil PAC, superiores a $50 \%$ logo abaixo dos $11 \mathrm{~cm}$ da superfície, crescendo para $86 \%$ nos horizontes Bt $\mathrm{e}$ Bt/Btx.
Os teores de $\mathrm{P}$ disponível variaram de 0 a $30 \mathrm{mg} \mathrm{dm}^{-3}$, com os maiores valores nos horizontes superficiais dos perfis cultivados. Esses resultados estão de acordo com a pobreza do material de origem e com os resultados encontrados por outros autores (Silva \& Ribeiro, 1992, Corrêa, 2005).

\section{Ataque sulfúrico}

De modo geral, observa-se aumento em profundidade dos elementos determinados após o ataque sulfúrico, acompanhando os teores de argila. Essa relação mostrou-se mais evidente nos Argissolos, uma vez que esses solos apresentam gradiente textural mais acentuado (Quadro 4).

Analisando os teores de $\mathrm{Fe}_{2} \mathrm{O}_{3}$, observou-se reduzido teor deste componente, com valores sempre abaixo de 2 dag $\mathrm{kg}^{-1}$. Tal fato deve-se à pobreza desse elemento no material de origem (Ribeiro, 1998).

Os menores teores de $\mathrm{Fe}_{2} \mathrm{O}_{3}$ no PAC são, provavelmente, consequência da maior restrição de drenagem deste perfill. Restrição de drenagem contribui para a intensificação da redução do $\mathrm{Fe}^{3+}$ para $\mathrm{Fe}^{2+}$, facilitando sua remoção por lixiviação (Schwertmann, 1985).

Quadro 3. Características químicas de rotina dos solos estudados ${ }^{(1)}$

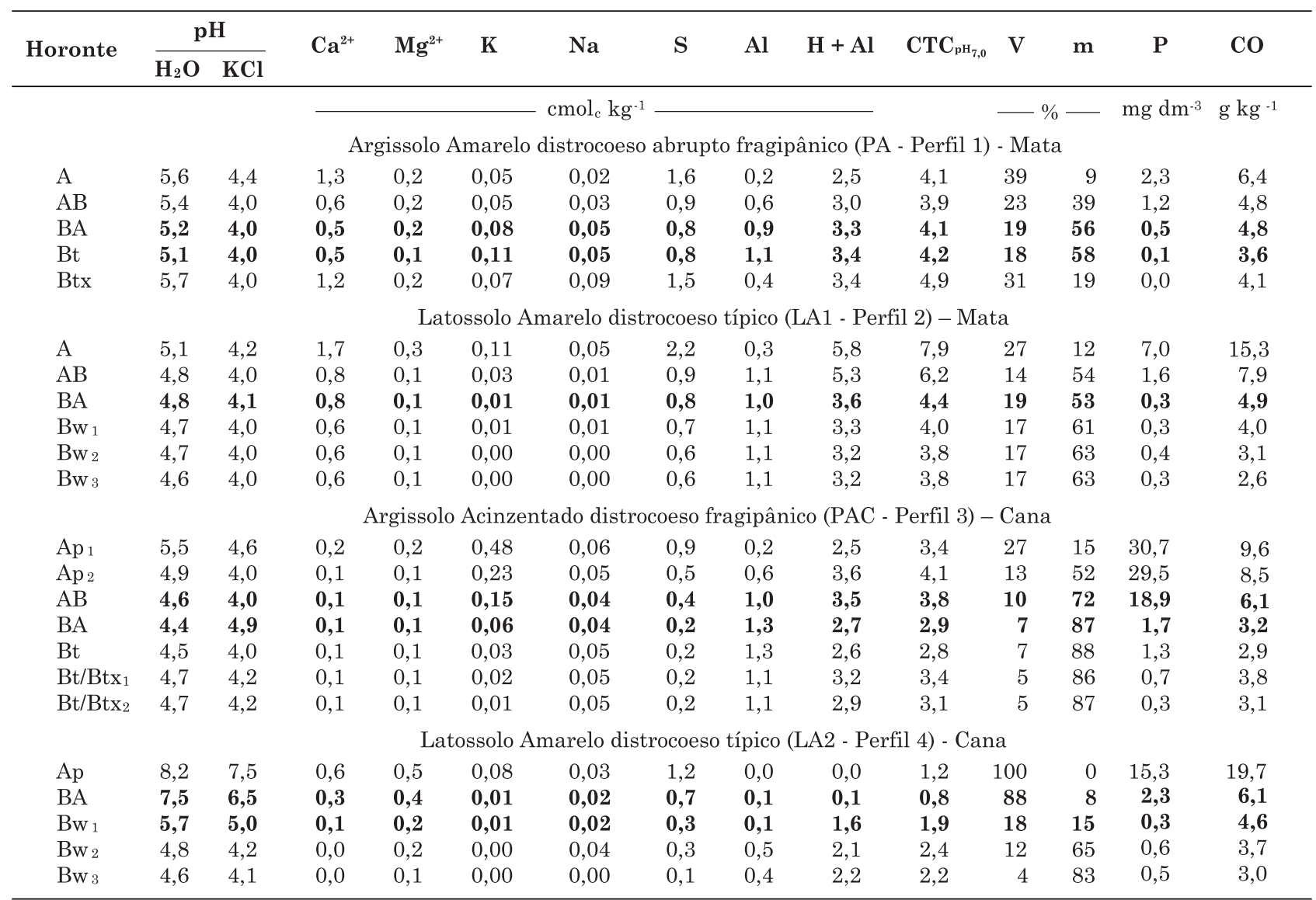

(1) Os resultados em negrito correspondem aos horizontes coesos identificados na descrição morfológica. 
Quadro 4. Resultados da digestão sulfúrica na TFSA e relações $\mathrm{Ki}, \mathrm{Kr}$ e $\mathrm{Al}_{2} \mathrm{O}_{3} / \mathrm{Fe}_{2} \mathrm{O}_{3}$ dos solos estudados

\begin{tabular}{|c|c|c|c|c|c|c|c|}
\hline Horizonte & $\mathrm{Fe}_{2} \mathrm{O}_{3}$ & $\mathrm{Al}_{2} \mathrm{O}_{3}$ & $\mathrm{SiO}_{2}$ & $\mathbf{P}_{2} \mathbf{O}_{5}$ & $\mathbf{K i}$ & $\mathbf{K r}$ & $\mathrm{Al}_{2} \mathrm{O}_{3} / \mathrm{Fe}$ \\
\hline & \multicolumn{7}{|c|}{ - dag kg-1 } \\
\hline & \multicolumn{7}{|c|}{ Argissolo Amarelo distrocoeso fragipânico (PA - Perfil 1) - Mata } \\
\hline A & 0,3 & 4,6 & 4,7 & 0,002 & 1,7 & 1,6 & 23,9 \\
\hline $\mathrm{AB}$ & 0,4 & 6,6 & 7,1 & 0,002 & 1,83 & 1,8 & 25,9 \\
\hline $\mathrm{BA}$ & 0,7 & 13,2 & 14,4 & 0,004 & 1,86 & 1,8 & 33,8 \\
\hline $\mathrm{Bt}$ & 1,0 & 20,5 & 19,1 & 0,004 & 1,59 & 1,5 & 35,7 \\
\hline \multirow[t]{2}{*}{ Btx } & 1,9 & 28,2 & 19,0 & 0,004 & 1,15 & 1,1 & 24,7 \\
\hline & \multicolumn{7}{|c|}{ Latossolo Amarelo distrocoeso típico (LA1 - Perfil 2) - Mata } \\
\hline $\mathrm{A}$ & 0,5 & 10,3 & 11,6 & 0,018 & 1,9 & 1,9 & 34,4 \\
\hline $\mathrm{AB}$ & 0,6 & 13,5 & 14,7 & 0,012 & 1,9 & 1,8 & 38,2 \\
\hline $\mathrm{BA}$ & 1,0 & 20,2 & 18,6 & 0,016 & 1,6 & 1,5 & 35,4 \\
\hline $\mathrm{Bw}_{1}$ & 1,0 & 21,9 & 19,0 & 0,015 & 1,5 & 1,4 & 36,5 \\
\hline $\mathrm{Bw}_{2}$ & 1,1 & 22,0 & 19,2 & 0,016 & 1,5 & 1,4 & 33,3 \\
\hline \multirow[t]{2}{*}{$\mathrm{Bw}_{3}$} & 1,4 & 26,8 & 19,5 & 0,016 & 1,2 & 1,2 & 33,3 \\
\hline & \multicolumn{7}{|c|}{ Argissolo Acinzentado distrocoeso fragipânico (PAC - Perfil 3) - Cana } \\
\hline $\mathrm{Ap}_{1}$ & 0,3 & 11,0 & 13,1 & 0,019 & 2,0 & 2,0 & 56,2 \\
\hline $\mathrm{Ap}_{2}$ & 0,3 & 11,9 & 13,8 & 0,019 & 2,0 & 1,9 & 61,0 \\
\hline $\mathrm{AB}$ & 0,4 & 15,8 & 18,2 & 0,012 & 2,0 & 1,9 & 70,8 \\
\hline $\mathrm{BA}$ & 0,4 & 18,1 & 20,6 & 0,001 & 1,9 & 1,9 & 72,0 \\
\hline $\mathrm{Bt}$ & 0,5 & 21,9 & 21,7 & 0,001 & 1,7 & 1,7 & 78,2 \\
\hline $\mathrm{Bt} / \mathrm{Btx}_{1}$ & 1,0 & 24,6 & 24,1 & 0,001 & 1,7 & 1,6 & 41,9 \\
\hline \multirow[t]{2}{*}{$\mathrm{Bt} / \mathrm{Btx}_{2}$} & 0,8 & 25,5 & 24,9 & 0,001 & 1,7 & 1,6 & 52,0 \\
\hline & \multicolumn{7}{|c|}{ Latossolo Amarelo distrocoeso típico (LA2 - Perfil 4) - Cana } \\
\hline Ap & 1,9 & 15,3 & 16,4 & 0,075 & 1,8 & 1,7 & 13,9 \\
\hline $\mathrm{BA}$ & 1,8 & 22,2 & 22,5 & 0,019 & 1,7 & 1,6 & 21,5 \\
\hline $\mathrm{Bw}_{1}$ & 2,0 & 25,7 & 24,8 & 0,019 & 1,6 & 1,6 & 21,9 \\
\hline $\mathrm{Bw}_{2}$ & 1,9 & 26,1 & 24,8 & 0,021 & 1,6 & 1,5 & 23,4 \\
\hline $\mathrm{Bw}_{3}$ & 1,9 & 27,1 & 25,9 & 0,021 & 1,6 & 1,6 & 24,5 \\
\hline
\end{tabular}

(1) Os resultados em negrito correspondem aos horizontes coesos identificados na descrição morfológica.

Além de apresentar os menores teores de Fe, o perfil PAC apresentou ainda as maiores densidades do solo. É citada na literatura a estreita relação entre o conteúdo de $\mathrm{Fe}_{2} \mathrm{O}_{3}$ e o grau de coesão, sendo os solos tão mais coesos e endurecidos quanto menores forem seus teores de $\mathrm{Fe}_{2} \mathrm{O}_{3}$ (Bennema \& Camargo, 1979). Segundo Ferreira et al. (1999), óxidos de Al e Fe e a matéria orgânica tendem a desorganizar as partículas do solo em escala microscópica, reduzindo a coesão. Corroborando tal afirmação, observa-se que a camada coesa que apresenta menor densidade do solo (1,46 kg dm $\mathrm{km}^{-3}$ ) (Perfil LA2) possui os maiores conteúdos de $\mathrm{Fe}_{2} \mathrm{O}_{3}$.

A natureza caulinítica da fração argila dos quatro perfis é compatível com valores da relação molecular $\mathrm{Ki}$, apresentando média de 1,7 , variando entre $1,1 \mathrm{e}$ 2,0. Tais resultados demonstram avançado estádio de intemperismo, estando coerente com outros autores (Moreau, 2001; Corrêa, 2005). O índice Ki de 1,7 ou maior é característico de mineralogia caulinítica (Embrapa, 1999).

Quanto à relação $\mathrm{Al}_{2} \mathrm{O}_{3} / \mathrm{Fe}_{2} \mathrm{O}_{3}$, houve tendência de aumento desta variável nos horizontes coesos dos solos estudados, principalmente no Argissolo Amarelo.

\section{CONSIDERAÇÕES FINAIS}

1. O caráter coeso foi identificado com exatidão pela morfologia nos horizontes subsuperficiais de Argissolos e Latossolos dos Tabuleiros Costeiros, mostrando-se mais pronunciado no Argissolo Acinzentado.

2. A morfologia e a densidade do solo, aliadas à determinação da superfície específica, mostraram-se eficientes na identificação dos horizontes coesos e não coesos dos Latossolos Amarelos e Argissolos da região dos Tabuleiros Costeiros.

3. O caráter coeso foi identificado em dois solos sob cultivo e dois solos sob vegetação nativa, o que sugere sua natureza pedogenética.

4. Os maiores valores da superfície específica observados nos horizontes coesos sugerem maior proporção de argilas muito finas nesses horizontes.

\section{AGRADECIMENTOS}

Ao $\mathrm{CNPq}$, pelo apoio financeiro e concessão de bolsas de Mestrado e Produtividade em Pesquisa. Às 
usinas Coruripe, Caeté, Marituba e Cachoeira, pelo apoio necessário na abertura das trincheiras e coleta dos solos.

\section{LITERATURA CITADA}

ARAÚJO FILHO, J.C.; CARVALHO, A. \& SILVA, F.B.R. Investigações preliminares sobre a pedogênese de horizontes coesos em solos dos tabuleiros costeiros do Nordeste do Brasil. In: WORKSHOP COESÃO EM SOLOS DOS TABULEIROS COSTEIROS, Aracaju, 2001 Anais. Aracaju, Embrapa Tabuleiros Costeiros, 2001. p.123-142.

BARROS, H.C.; DRUMONT, J.L.; CAMARGO, M.N.; LEMOS, P.O.C. \& LEMOS, R.C.; MENDES, W. Levantamento de reconhecimento dos solos do Estado do Rio de Janeiro e Distrito Federal. Rio de Janeiro, Ministério da Agricultura, 1958. 350p. (SNPA-Boletim Técnico, 11)

BARZEGAR, R.; RENGASAMY, P. \& OADES, M. Effects of clay type and rate of wetting on the mellowing of compacted soils. Geoderma, 68:39-49, 1995.

BENNEMA, J. \& CAMARGO, M.N. Some remarks on Brazilian Latosols in relation to the Oxisols of soil taxonomy. In: INTERNATION SOIL CLASSIFICATION WORKSHOP, 2., Malaysia, 1978. Proceedings. Bangkok, SSD - LDD, 1979. p.233-235.

CORRÊA, M.M. Atributos físicos, químicos, mineralógicos e micromorfológicos de solos e ambiente agrícola nas várzeas de Sousa - PB. Viçosa, MG, Universidade Federal de Viçosa, 2000. 107p. (Tese de Mestrado)

CORRÊA, M.M. Gênese de horizontes coesos e fragipãs de solos do ambiente Tabuleiros Costeiros. Viçosa, MG, Universidade Federal de Viçosa, 2005. 72p. (Tese de Doutorado)

CORRÊA, M.M.; ANDRADE, F.V.; MENDONÇA, E.S.; SHAEFER, C.E.G.R.; PEREIRA, T.T.C. \& ALMEIDA, C.C. Ácidos orgânicos de baixo peso molecular, ácidos húmicos e alterações em algumas propriedades físicas e químicas de Latossolos, Plintossolo e Neossolo Quartzarênico. R. Bras. Ci. Solo, 32:121-131, 2008.

EMPRESA BRASILEIRA DE PESQUISA AGROPECUÁRIA EMBRAPA. Serviço Nacional de Levantamento e Conservação de Solos. Levantamento de reconhecimento dos solos do estado do Espírito Santo. Rio de Janeiro, 1978. 461p. (Boletim técnico, 45)

EMPRESA BRASILEIRA DE PESQUISA AGROPECUÁRIA . EMBRAPA. Centro Nacional de Pesquisa de Solos. Manual de métodos de análise de solo. 2.ed. Rio de Janeiro, 1997. 212p. (Documentos, 1)

EMPRESA BRASILEIRA DE PESQUISA AGROPECUARIA EMBRAPA. Centro Nacional de Pesquisa de Solos. Sistema brasileiro de classificação de solos. Brasília, 1999. $412 \mathrm{p}$.

EMPRESA BRASILEIRA DE PESQUISA AGROPECUÁRIA EMBRAPA. Centro Nacional de Pesquisa de Solos. Sistema brasileiro de classificação de solos. Brasília, 2006. $306 \mathrm{p}$.
FERREIRA, M.M.; FERNANDES, B. \& CURI, N. Mineralogia da fração argila e estrutura de Latossolos da Região Sudeste do Brasil. R. Bras. Ci. Solo, 23:507-514, 1999.

GIAROLA, N.F.B.; SILVA, A.P.; TORMESA, C.; SOUZA, L.S \& RIBEIRO, L.P. Similaridade entre o caráter coeso dos solos e o comportamento hardsetting: Estudo de caso. R. Bras. Ci. Solo, 25:239-247, 2001.

JACOMINE, P.K.T. Distribuição geográfica, características e classificação dos solos coesos dos Tabuleiros Costeiros. In: REUNIÃO TÉCNICA SOBRE SOLOS COESOS DOS TABULEIROS, Cruz das Almas, 1996. Anais. Aracaju, Embrapa Tabuleiros Costeiros, 1996. p.13-24.

JACOMINE, P.K.T.; MONTENEGRO, J.O. \& RIBEIRO, M.R. Levantamento exploratório-reconhecimento de solos do Estado de Sergipe. Recife, Empresa Brasileira de Pesquisa Agropecuária, 1975. 506p. (Boletim Técnico, 36. Série Recursos de Solos, 6)

JACKSON, M.L. Soil chemical analysis: Advanced course. Madison, Prentice-Hall, 1979. 895p.

MENDES JÚNIOR, B.O. Perfil econômico de Alagoas. Fortaleza, Banco do Nordeste, 2002. 58p.

MOREAU, A.M.S.S.; KER, J.C.; COSTA, L.M. \& GOMES, F.H. Caracterização de solos de duas topossequências em tabuleiros costeiros do sul da Bahia. R. Bras. Ci Solo, 30:1007-1019, 2006.

MOREAU, A.M.S.S. Gênese, mineralogia e micromorfologia de horizontes coeso, fragipã e duripã em solos do Tabuleiro Costeiro no Sul da Bahia. Viçosa, MG, Universidade Federal de Viçosa, 2001. 138p. (Tese de Doutorado)

MULLINS, C.E. Hardsetting. In: LAL, R.; BLUM, W.H.; VALENTINE, C. \& STEWART, B.A., eds. Methods for assesment of soil degradation. Advances in soil science. New York, CRC Press, 1997. p.109-128.

PETERSEN, G.W.; RANNEY. R.W.; CUNNINGHAM, R.L. \& MATELSKI R.P. Fragipans in Pennsylvania soils: A statistical study of laboratory data. Soil Sci. Soc. Am. Proc., 34:719-722, 1970

QUIRK, J.P. Significance of surface areas calculated from water vapour sorption isotherms by the use of BET equation. Soil Sci., 80:423-430, 1955.

REZENDE, J.O. Solos coesos dos Tabuleiros Costeiros: Limitações agrícolas e manejo. Salvador, SEAGRI, 2000. 117p. (Série Estudos Agrícolas, 1)

RIBEIRO, L.P. Os Latossolos Amarelos do Recôncavo Baiano: Gênese, evolução e degradação. Salvador, Seplantec, CADCT, 1998. 99p.

RIBEIRO, M.R. Características morfológicas dos horizontes coesos dos solos dos Tabuleiros Costeiros. In: WORKSHOP COESÃO EM SOLOS DOS TABULEIROS COSTEIROS, Aracaju, 2001. Anais. Aracaju, Embrapa Tabuleiros Costeiros, 2001. p.161-168.

RICHIE, A.; WILDING, L.P.; HALL, G.F. \& STAHNKE, C.R. Genetic implications of B horizons in Aqualfs of Northeastern Ohio. Soil Sci. Soc. Am. J., 38:351-358, 1974.

SANTOS, R.D.; LEMOS, R.C.; SANTOS, H.G.; KER, J.C. \& ANJOS, L.H. Manual de descrição e coleta de solos no campo. 5.ed. Viçosa, MG, Sociedade Brasileira de Ciência do Solo, 2005. 100p. 
SCHWERTMANN, U. The effect of environments on iron oxide minerals. Adv. Soil Sci., 1:172-200, 1985.

SILVA, M.S.L. \& RIBEIRO, M.R. Influência do cultivo contínuo da cana-de-açúcar em propriedades morfológicas e físicas de solos argilosos de tabuleiro no Estado de Alagoas. R. Bras. Ci. Solo, 16:397-402, 1992.

SILVA, A.J.N. Caracterização de Latossolos Amarelos sob cultivo contínuo de cana-de-açúcar no Estado de Alagoas. Recife, Universidade Federal Rural de Pernambuco, 1996. 133p. (Tese de Mestrado)

SILVA, A.J.N. \& RIBEIRO, M.R. Caracteraização de Latossolo Amarelo sob cultivo contínuo de cana-de-açúcar no Estado de Alagoas: Propriedades químicas. R. Bras. Ci. Solo, 22:291-299, 1998.
SILVA, M.S.L.; KLAMT, E.; CAVALCANTI, A.C. \& KROTH, P.L. Adensamento subsuperficial em solos do semi-árido: Processos geológicos e, ou, pedogenéticos. R. Bras. Eng. Agric. Amb., 6:314-320, 2002.

SOUZA, L.S.; SOUZA, L.D. \& CALDAS, R.C. Identificação da coesão com base em atributos físicos convencionais em solos dos Tabuleiros Costeiros. In: WORKSHOP COESÃO EM SOLOS DOS TABULEIROS COSTEIROS, Aracaju, 2001. Anais. Aracaju, Embrapa Tabuleiros Costeiros, 2001. p.169-190.

SOUZA, L.S. Uso e manejo dos solos coesos dos Tabuleiros Costeiros. In: REUNIÃO TÉCNICA SOBRE SOLOS COESOS DOS TABUlEIROS COSTEIROS, Cruz das Almas, 1996. Anais. Aracaju, Embrapa Tabuleiros Costeiros, 1996. p.36-75. 
José de Almeida Lima Neto et al. 